\title{
Neurosurgeons on the front line: experience from the center of the storm in Italy
}

\author{
Claudio Bernucci, MD, ${ }^{1}$ Andrea Fanti, MD, ${ }^{1,2}$ Pierlorenzo Veiceschi, MD, ${ }^{2,3}$ Emanuele Costi, MD, ${ }^{1}$ \\ Angelo Mirco Sicignano, MD, ${ }^{1}$ and Carlo Brembilla, MD1
}

\begin{abstract}
1Department of Neurosurgery, ASST Papa Giovanni XXIII Hospital, Bergamo; ${ }^{2}$ Department of Neurosurgery, Scuola di Specializzazione in Neurochirurgia, Università degli Studi di Pavia; and ${ }^{3}$ Department of Neurosurgery, Scuola di Specializzazione in Neurochirurgia, Università degli Studi dell'Insubria, Varese, Italy

In this tumultuous time, the entire world has been shaken up by the COVID-19 outbreak. Italy has had one of the highest infection-related mortality rates. Bergamo, a city in eastern Lombardy, was among the most affected. Here, the authors describe the main healthcare actions taken at their institution to stem the crisis, with particular concern regarding the fate of their neurosurgery department. Among the different topics, the authors particularly focus on the retraining of neurosurgeons, organization of activities, and what should be the role of neurosurgeons during a pandemic.

https://thejns.org/doi/abs/10.3171/2020.9.FOCUS20577
\end{abstract}

KEYWORDS neurosurgery; coronavirus; COVID-19; SARS-CoV-2; pandemic

$\mathrm{S}$ TARTING in the last weeks of February 2020, Italy was struck by a massive wave of infections due to severe acute respiratory syndrome coronavirus 2 (SARSCoV-2) coming from China. ${ }^{1}$ This emergency led to 1) the application of many containment measures with several political acts and 2) the implementation of urgent reforms within the national healthcare system. ${ }^{2}$ Hospitals and newly dedicated facilities were established to treat patients affected by COVID-19. Lombardy was the Italian region most hit by the pandemic, and here the standard of care was reduced dramatically, affecting nonsurgical and surgical activities equally. ${ }^{3}$

Beginning March 8, 2020, there was a complete conversion of the neurosurgical regional health organization in a hub-and-spoke system, following both capacity and geographical criteria. ${ }^{4}$ Three hub centers located in Milan, Brescia, and Varese were established for emergencies, 5,6 while another center in Milan was chosen to deal only with oncological cases. ${ }^{7}$ All other neurosurgery departments in Lombardy, as spoke centers, focused almost completely on COVID-19 patients.

Papa Giovanni XXIII Hospital in Bergamo, given its large capacity, became particularly active in the face of the epidemic. Most neurosurgeons from this hospital were involved in the treatment of COVID-19 patients, while the remaining neurosurgeons dealt with patients with neurosurgical pathologies in temporary surgical wards.

The aim of this paper is to share our significant experi- ence as a neurosurgical spoke center, hoping that it can be helpful to other colleagues in the world, in the distant event of a new pandemic.

\section{Neurosurgeons Assigned to COVID-19 Wards Papa Giovanni XXIII Hospital in Bergamo: The Eye of the Storm}

During the COVID-19 pandemic, the emergency department at our hospital was overcrowded by patients (Fig. 1), with a maximum of 90 patients daily in the 2 nd week of March; most of them were admitted to wards or dedicated ICUs. ${ }^{8}$

Starting on February 22, 2020, the Department of Infectious Diseases totally focused on COVID-19 patient care, and, in less than a week, all 48 beds were occupied. Several surgical and medical wards were progressively cleared out and converted into COVID-19 facilities that became occupied by patients needing ventilatory support through continuous positive airway pressure; up to 140 noninvasive ventilation (NIV) devices were working simultaneously. At the end of the 1st week of March, 5 wards dedicated to COVID-19 patients were activated and dealt with 48 patients each. This required a significant amount of resources, including healthcare workers, but also medical supplies of all kinds (e.g., masks, protective equipment, ventilators, oxygen, and medication).

The cumulative number of ICU and semi-ICU beds

ABBREVIATIONS NIV = noninvasive ventilation; OR = operating room; PPE = personal protective equipment; SARS-CoV-2 = severe acute respiratory syndrome coronavirus 2. 


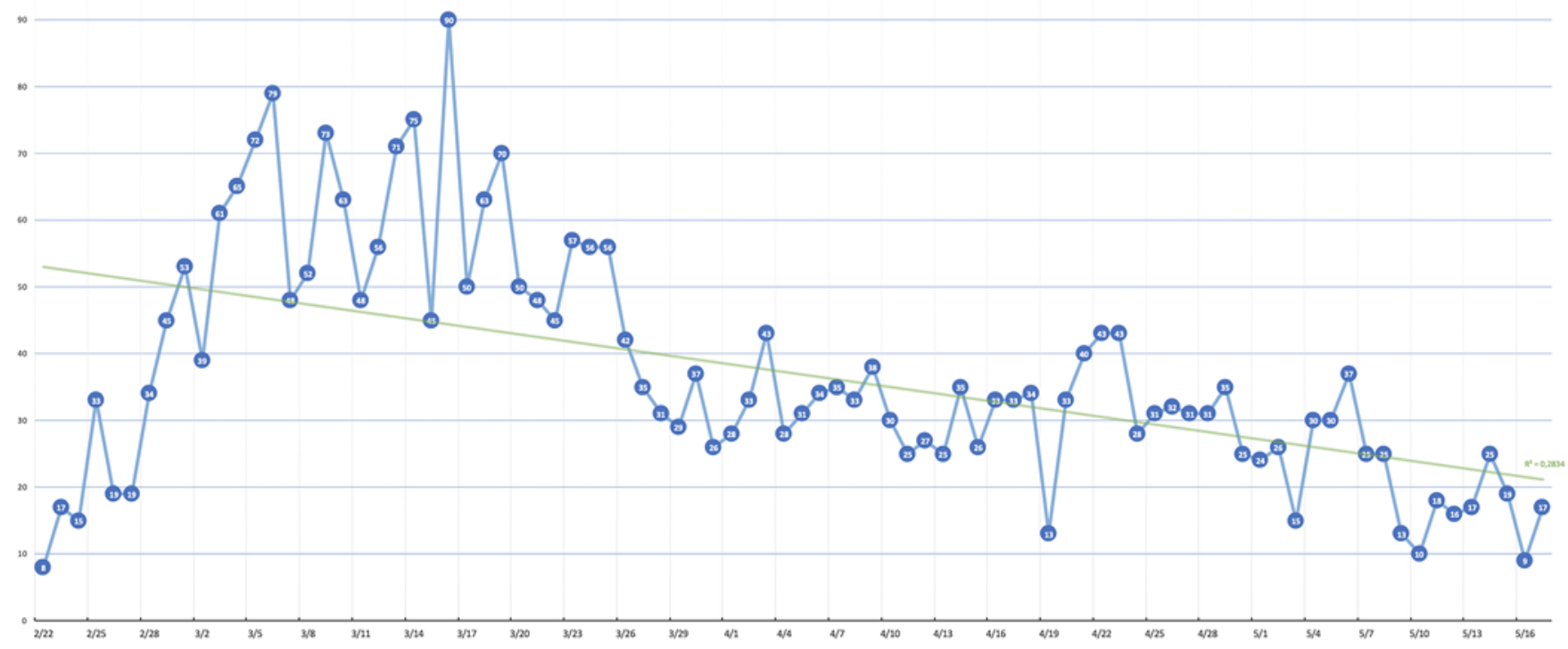

FIG. 1. Access list for COVID-19 in the emergency department of Papa Giovanni XXIII Hospital in Bergamo, between February 22 and May 17, 2020. The maximum daily number was reached on March 17, followed by a slow and progressive reduction in cases.

increased from 93 beds (53 in the ICU and 40 in stepdown units) beds to 100 ( 88 in the ICU and 12 in stepdown units). Therefore, there was actually a great increase in ICU capacity, since 25 stepdown unit beds were converted to ICU beds. On March 28, COVID-19 patients occupied 498 of 779 hospital total beds; 92 of them were admitted to a COVID-19 ICU, making Bergamo one of the worst hit cities worldwide.

\section{How Do Neurosurgeons Train to Treat Patients Affected by COVID-19?}

Since all available forces were needed, physicians from various medical and surgical specialties were recruited and redeployed. ${ }^{9}$ Once COVID-19 units were activated, nearly $35 \%$ of the hospital personnel (more than 300 doctors and 500 nurses) were reallocated ${ }^{8}$ Considering their very basic or even insufficient knowledge and skills in pulmonology, infectious disease, and even critical care topics, it was necessary to start rapid, intensive, and effective staff training.

Beginning on March 6, 2020, training courses were established for doctors, paramedics, and all volunteers involved in the health emergency. Until April 4, more than 4200 participants among approximately 4350 hospital workers attended a total of 59 training sessions. These sessions were organized by the hospital health management, and the teachers were specialists selected according to the topic of the course (e.g., pulmonologists, anesthesiologists, specialized nurses, hygienists, and infectious disease specialists). The courses were scheduled in weekly repeatable modules, each lasting nearly 2 hours and involving the following technical and security topics:

- Clinical, diagnostic, and treatment aspects of COVID-19;
- Ventilatory support: devices and management of invasive and NIV;

- Protective measures against the infection;

- Correct usage of personal protective equipment (PPE; e.g., masks, gloves, gowns, ventilators, and filters for ventilators);

- Information technology (informatics procedures and medical paperwork);

- Hygienic and sanitary standards in COVID-19-free and COVID-19-positive areas.

\section{Therapeutic Guidelines for Univocally Treating Patients With COVID-19}

The hospital committee established a website accessible by every computer, containing specific instructions and constantly updated guidelines. These guidelines regarded the following:

- General help (list of on-call pneumologists or anesthesiologists, access to clinical studies);

- Therapeutic guidelines (standard treatment, flowcharts of oxygen administration, management of associated pathologies such as thrombosis, hyperglycemia, nasogastric feeding, and NIV weaning);

- Diagnostic guidelines (methods of swab collections, criteria to request radiological examinations);

- Operative video (how medical instruments such as NIV are used, protective measures);

- Bureaucratic acts (infective disease notification, cadaver administration, funerary activities);

- Out-of-hospital services (patient discharge criteria, instruction for solitary confinement).

\section{Performance of Grand Rounds in the COVID-19 Wards}

In the hospital, many wards were entirely converted to 
HOSPITAL GENERAL PLAN

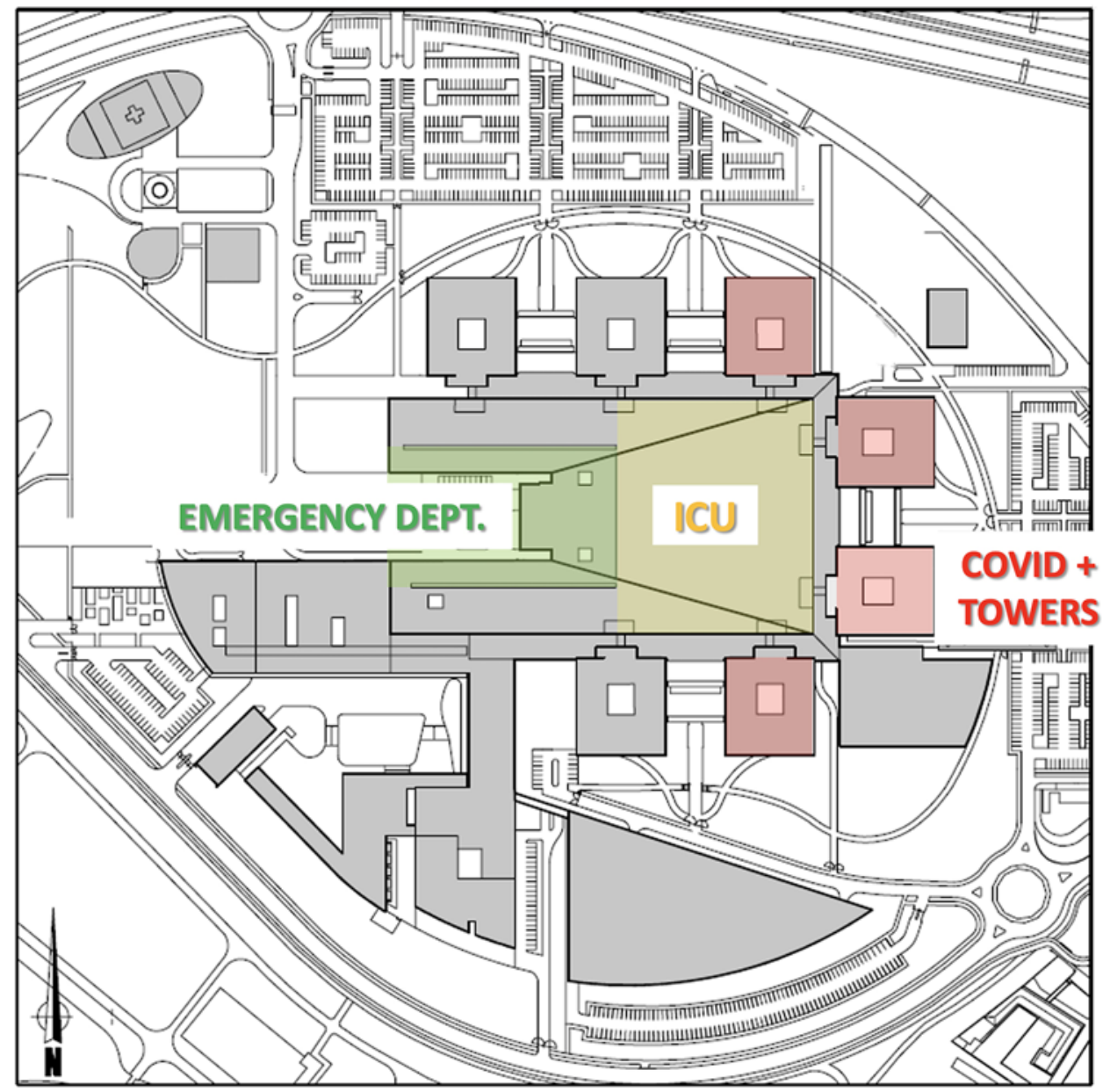

FIG. 2. General plan at the Papa Giovanni XXIII Hospital. As described in the text, 4 of the 7 towers were dedicated to COVID-19 patients.

focus on patients with COVID-19. Those included the Department of Infectious Diseases, the totality of Tower 4 (hematology, pneumology, and gastroenterology services), and part of Tower 3 (only the Department of Neurology). The overall number of beds inside the COVID-19 wards was nearly 400, with an additional 92 COVID-19 patients in the ICU (Fig. 2). ${ }^{4}$

The clinical activity was divided into three rotations: a morning shift from 8 AM to $3 \mathrm{PM}$, an afternoon session from 3 PM to 10 PM, and a night shift from 10 PM to $8 \mathrm{AM}$. Two physicians took care of an average of 20-26 patients during each shift.

The assignments were managed by combining surgeons and clinicians in the same rotation to help the surgeons and those less familiar with pulmonary pathologies or internal medicine. However, all recruited specialists, including neurosurgeons, were involved directly in patient care during each shift. In the event of difficult cases or cardiorespiratory emergencies, pulmonologists and anesthesiologists were available 24 hours a day.

COVID-19 wards were divided into two areas, a clean area and a contaminated area, which were delimited by a red strip on the floor (Fig. 3). At the beginning of the shift, the physicians whose shift just ended provided information about the patients at handoff. By agreement, one doctor dressed in protective equipment (surgical scrub, N95 mask, double gloves, cuff, and glasses or visor) and moved to the contaminated area to treat each patient. The other one stayed in the clean area writing clinical parameters, prescribing therapy according to the needs, or asking for radiological examinations or medical advice. We chose this method because of the necessity to reduce PPE use and the risk of contamination. After undressing and being accurately sanitized, the first doctor reached the colleague in the clean area. Since every patient was isolated in his or her room, relatives were informed daily by the medical staff about the patient's health conditions. Thereafter, a video service was established for patients hospitalized in the COVID-19 wards and ICU, keeping patients in touch with their loved ones.

Among the various services available, the Department of Psychiatry offered constant psychological support for healthcare workers, with the project "Taking care of those who take care." 


\section{FLOOR ORGANIZATION OF A COVID WARD}

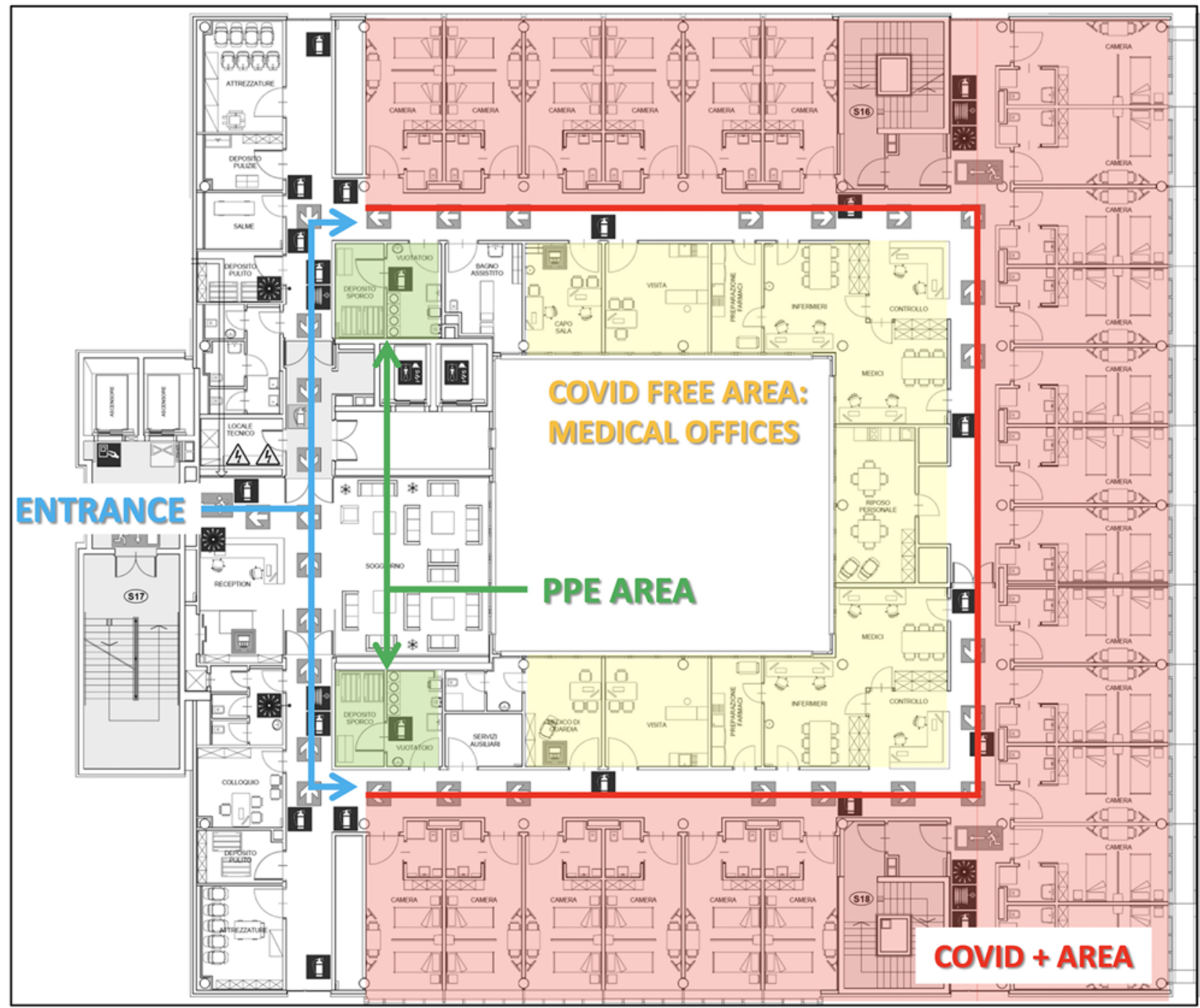

FIG. 3. Typical organization of a COVID-19 ward inside a hospital tower.

\section{Neurosurgeons Not Assigned to COVID-19 Wards}

The recruitment of neurosurgical staff started in the 1st week of March and gradually increased. On March 20, 75\% of our total staff was assigned to COVID-19 wards. Surgeons from our team were selected based on the individual risk stratification to reduce complications in case of contagion.

We considered major risk factors such as obesity, age older than 60 years, and chronic diseases (e.g., diabetes mellitus, asthma, and systolic hypertension). Neurosurgeons not assigned to COVID-19 wards continued to assist neurosurgical patients, with several differences from normal practice.

\section{How Has the Neurosurgery Department Been Reorganized?}

Before the emergency, the neurosurgery department included 34 regular beds and another 16 dedicated positions in two different ICUs. Since the majority of the staff was involved treating COVID-19 patients, the neurosurgeon on-call presence ( 24 hours a day, 7 days a week) was suspended and replaced with an availability of 24 hours a day, 7 days a week.

The number of available beds in the ward was initially reduced and moved to another floor, as happened in the majority of the surgical subspecialties. The lack of available beds in the ICU for neurological patients, the shift of neurosurgical personnel in COVID-19 wards, and the unavailability of endovascular treatment together redesigned the normal routine. Since our hospital was appointed as a spoke facility, the Lombard Emergency Service directed patients to the other regional hubs. Nevertheless, neurosurgical patients continued to present to our emergency department. Based on the urgency and the requirement of an ICU bed for the condition at hand, ${ }^{10}$ these patients were ad- 
mitted to the temporary ward or sent to a designated neurosurgical hub. Unstable patients were initially stabilized and then sent to another hub for major surgery (e.g., a patient requiring immediate external ventricular drainage system placement and then being sent to the hub for aneurysm treatment). These decisions belonged to the neurosurgeons not assigned to the COVID-19 wards. Patients with malignant tumors were sent to the neurooncological hub and were usually treated by the referring neurosurgeons in a cooperative spirit between hub-and-spoke colleagues. The outpatient clinic was maintained only for urgent consultations, and all other visits were cancelled. When possible, patients were managed via telephone, and their neuroradiological images were reviewed via the internet. ${ }^{11}$

\section{Surgical Activity During the COVID-19 Era}

The COVID-19 pandemic had profound implications on our surgical activities. We compared the first 2 months after the institution of the hub-and-spoke system in Lombardy with the corresponding months of 2019.

In March and April 2019,251 total surgical procedures were accomplished; 200 (80\%) of them were elective, 12 $(5 \%)$ were emergency surgeries (performed as quickly as possible and ideally within 1 hour), and 39 (16\%) were urgent surgeries (performed within 4-10 hours). One hundred nine (43\%) were cranial, 119 (47\%) were spinal, and $23(9 \%)$ involved peripheral nerve surgery.

In March and April 2020, only 47 interventions were performed; 15 (32\%) of them were elective, 9 (19\%) were emergency, and 23 (49\%) were urgent. Twenty-five (53\%) were cranial and $22(47 \%)$ were spinal.

Compared with the past year, surgical activities suffered a dramatic decrease, accounting for a statistically significant overall reduction rate of $81.3 \%$ ( $\mathrm{p}=0.0001$ according to Fisher's exact test). After March 1, the number of elective surgeries performed was reduced each week, and after the 3rd week of March, no more elective surgeries were performed (Table 1). In fact, from March 15 to the end of April, only urgent or emergency surgeries were performed. At the very beginning, neurosurgical patients were not routinely tested for SARS-CoV-2 infection because the full emergency had not yet been declared and dedicated laboratories had not been established. After March 12, at admission to the emergency department, all patients underwent nasopharyngeal swabbing (real-time polymerase chain reaction) and chest radiography to check for signs of interstitial pneumonia. If the results of the swab test were positive, or if the chest radiograph was suggestive of COVID-19 (even despite a negative swab), the patients were further examined with chest CT scanning. If emergency neurosurgical intervention was required, whether patients had positive test results or the results were unknown (swab result still ongoing), the surgical team took special precautions in the operating room. ${ }^{12,13}$ From March 12 to the end of April, we operated on 4 COVID-19-positive and 22 COVID-19-negative patients.

\section{Resumption of Activities}

During the last weeks of April, there was a progressive reduction of new infections in the general population; hos- pitalizations in the COVID-19 wards and ICU decreased, with more daily recoveries and discharges. On May 4, 2020, the Italian government decreed so-called phase 2, indicating a cautious and progressive reduction of the previously established global lockdown. Healthcare activities benefitted from this lessening of the stricter rules in place during the COVID-19 outbreak.

\section{Neurosurgical Ward}

Between March 22 and the end of April, the multidisciplinary common surgical ward was split into two floors of Tower 3: one for COVID-19-positive surgical patients and the other for COVID-19-negative patients. Nasal swabs were routinely administered on admission to the emergency department, as described previously, and patients needing to be admitted were kept inside "gray zones" until the test result was available. After that, they were hospitalized in the dedicated sector. Inside the COVID-19-positive surgical department, the precautions taken by healthcare workers and the general floor organization were quite similar to those of the COVID-19 wards. To reduce the risk of contagion, no visitors were allowed in the departments, and adequate PPE was utilized by healthcare workers. Since most COVID-19 wards were closed, beginning on April 27, all neurosurgeons on our team assigned to COVID-19 wards were freed from this burden, and they returned to their regular activity. Starting from the 1st week of May, the neurosurgical ward finally returned to the original floor, even with a reduced number of beds compared with the pre-COVID-19 era (18 vs 30 beds, respectively).

\section{Outpatient Clinic}

Outpatient visits were also remodeled according to the emergency. By March 6, these visits had been reduced to one weekly session for emergencies and a second for oncological cases. Starting on May 4, the outpatient clinic was reopened in a downscaled capacity, by performing triage on arrival and reducing the number of patients who visited every day. The waiting room was also rearranged to avoid the gathering of patients at arrival. Accurate sanitation of the outpatient environment was performed between one visit and the next.

\section{Operating Rooms}

Starting May 5, there was a cautious resumption of elective neurosurgical activities. At the very beginning, only 2 weekly neurosurgical slots were assigned to our department. We progressively increased our surgical activity, and, starting on May 25, we had 10 weekly surgical slots. In June 2020, we reached 50\% of the pre-COVID-19 regular surgical activity. All patients were tested with a nasopharyngeal swab within 72 hours before surgery. If the test results were positive, surgery was postponed; if it was necessary, they were moved to dedicated COVID-19 operating rooms. In parallel, there was a significant increase in emergency surgical sessions. This was probably related to the reopening of social and working spaces once phase 2 was decreed.

\section{Epidemiological Surveillance Among Healthcare Workers}

Medical and nonmedical personnel represented the ac- 
TABLE 1. List of surgical operations performed during March and April 2020

\begin{tabular}{|c|c|c|c|c|c|}
\hline Case No. & Age (yrs), Sex & Date* $^{*}$ & Surgery & Diagnosis & Intervention \\
\hline 1 & $48, F$ & $03 / 01$ & Emergency & Obstructive hydrocephalus & EVD \\
\hline 2 & $48, M$ & $03 / 02$ & Elective & Brain tumor & Microsurgical removal \\
\hline 3 & $53, \mathrm{~F}$ & $03 / 02$ & Elective & Cervical myelopathy & Anterior cervical discectomy \& fusion \\
\hline 4 & $72, \mathrm{M}$ & $03 / 02$ & Elective & Lumbar stenosis & Posterior decompression \\
\hline 5 & $74, \mathrm{~F}$ & $03 / 03$ & Elective & Spinal neurinoma & Microsurgical removal \\
\hline 6 & $42, \mathrm{~F}$ & $03 / 03$ & Elective & Lumbar disc herniation & Microdiscectomy \\
\hline 7 & $51, \mathrm{M}$ & $03 / 03$ & Elective & Lumbar disc herniation & Microdiscectomy \\
\hline 8 & $59, \mathrm{~F}$ & $03 / 04$ & Elective & Lumbar disc herniation & Microdiscectomy \\
\hline 9 & $30, \mathrm{M}$ & $03 / 04$ & Elective & Lumbar disc herniation & Microdiscectomy \\
\hline 10 & $69, M$ & $03 / 04$ & Elective & Recurrent lumbar herniation & Posterior lumbar interbody fusion \\
\hline 11 & $72, \mathrm{M}$ & $03 / 05$ & Elective & Lumbar synovial cyst & Microsurgical removal \\
\hline 12 & $66, F$ & $03 / 05$ & Elective & Brain metastasis & Microsurgical removal \\
\hline 13 & $50, M$ & $03 / 05$ & Elective & Lumbar disc herniation & Microdiscectomy \\
\hline 14 & $81, M$ & $03 / 05$ & Elective & Chronic subdural hemorrhage & Burr hole \\
\hline 15 & $31, \mathrm{M}$ & $03 / 07$ & Urgent & Chronic subdural hemorrhage & Burr hole \\
\hline 16 & $59, F$ & $03 / 08$ & Urgent & Obstructive hydrocephalus & EVD \\
\hline 17 & $53, \mathrm{M}$ & $03 / 08$ & Urgent & Diffuse cerebral edema & Decompressive craniectomy \\
\hline 18 & $61, \mathrm{~F}$ & $03 / 09$ & Urgent & Thoracic fracture & Decompression \& fusion \\
\hline 19 & $72, \mathrm{~F}$ & $03 / 10$ & Elective & Cerebral meningioma & Microsurgical removal \\
\hline 20 & $28, M$ & $03 / 11$ & Elective & Intraaxial brain tumor & Microsurgical removal \\
\hline 21 & $39, \mathrm{~F}$ & $03 / 12$ & Urgent & Cauda equina syndrome & Microdiscectomy \\
\hline 22 & $69, \mathrm{M}$ & $03 / 15$ & Urgent & Postsurgical liquorrhea & Revision \\
\hline 23 & $69, F$ & $03 / 30$ & Urgent & Epidural spinal access & Microsurgical drainage \\
\hline 24 & $75, M$ & $03 / 30$ & Urgent & Chronic subdural hemorrhage & Burr hole \\
\hline 25 & $33, \mathrm{~F}$ & $03 / 31$ & Emergency & Acute subdural hemorrhage & Decompressive craniectomy \\
\hline 26 & $33, F$ & $03 / 31$ & Emergency & Diffuse cerebral edema & Decompressive craniectomy \\
\hline 27 & $38, M$ & $03 / 31$ & Urgent & Acute extradural hemorrhage & Burr hole \\
\hline 28 & $75, \mathrm{M}$ & $04 / 01$ & Emergency & Acute subdural hemorrhage & Decompressive craniotomy \\
\hline 29 & $31, \mathrm{M}$ & $04 / 01$ & Urgent & Thoracic fracture & Vertebroplasty + fusion \\
\hline 30 & $78, M$ & $04 / 02$ & Urgent & Chronic subdural hemorrhage & Burr hole \\
\hline 31 & $57, \mathrm{M}$ & $04 / 05$ & Emergency & SAH due to ACoA aneurysm & EVD, transferred to regional hub \\
\hline 32 & $85, \mathrm{M}$ & $04 / 14$ & Urgent & Chronic subdural hemorrhage & Burr hole \\
\hline 33 & $82, F$ & $04 / 14$ & Urgent & Chronic subdural hemorrhage & Burr hole \\
\hline 34 & $85, \mathrm{M}$ & $04 / 16$ & Urgent & Chronic subdural hemorrhage & Burr hole \\
\hline 35 & $79, \mathrm{M}$ & $04 / 16$ & Urgent & Chronic subdural hemorrhage & Burr hole \\
\hline 36 & $31, \mathrm{M}$ & $04 / 19$ & Urgent & Chronic subdural hemorrhage & Burr hole \\
\hline 37 & $56, \mathrm{M}$ & $04 / 20$ & Urgent & Lumbar spondylosis & Decompression \\
\hline 38 & $55, \mathrm{M}$ & $04 / 21$ & Emergency & SAH due to ACoA aneurysm & EVD + endovascular therapy \\
\hline 39 & $57, \mathrm{M}$ & $04 / 24$ & Urgent & Cauda equina syndrome & Microdiscectomy \\
\hline 40 & $45, \mathrm{~F}$ & $04 / 24$ & Emergency & Acute subdural hemorrhage & Decompressive craniectomy \\
\hline 41 & $59, \mathrm{M}$ & $04 / 25$ & Emergency & SAH due to ACoA aneurysm & EVD + endovascular therapy \\
\hline 42 & $29, \mathrm{~F}$ & $04 / 27$ & Emergency & SAH + IVH due to rt frontal AVM & EVD + endovascular therapy \\
\hline 43 & $50, \mathrm{M}$ & $04 / 27$ & Urgent & Cauda equina syndrome & Posterior decompression \\
\hline 44 & $53, \mathrm{M}$ & $04 / 28$ & Urgent & Cauda equina syndrome & Posterior decompression \\
\hline 45 & $78, \mathrm{~F}$ & $04 / 29$ & Urgent & Cervical myelopathy & Open-door cervical decompression \\
\hline 46 & $41, M$ & $04 / 29$ & Urgent & Traumatic cervical disc herniation & Anterior cervical discectomy \& fusion \\
\hline 47 & $61, \mathrm{M}$ & $04 / 30$ & Urgent & Neurostimulator infection & Revision surgery \\
\hline
\end{tabular}

$\mathrm{ACOA}=$ anterior communicating artery; $\mathrm{AVM}=$ arteriovenous malformation; $\mathrm{EVD}=$ external ventricular drainage; IVH = intraventricular hemorrhage; $\mathrm{SAH}=$ subarachnoid hemorrhage.

${ }^{*}$ Presented as month/day. 
tive frontline against the virus, and special surveillance was undertaken to counteract infection spread among these individuals. To avoid the possibility of these personnel becoming asymptomatic virus carriers, many prophylaxis measures were put in place. First, all staff had an obligation to measure their body temperature on arrival at the hospital. A nasal swab for virus research was performed in personnel with typical symptoms or fever or in case they had unsafe contact with possibly positive or positive patients. When serological tests became available, hospital workers (medical, nursing, and auxiliary staff) were screened for active or previous infection. During the first sample, 1524 tests were performed at the hospital. According to the seroprevalence studies up to May 15, $25.6 \%$ of tests had been positive, $72 \%$ had been negative, and $2.4 \%$ were possibly positive. Within the neurosurgery medical staff, considering 12 attending physicians and 3 residents, the positive rate was $26 \%(4 / 15)$.

\section{Discussion}

Since the new coronavirus disease, referred to as COVID-19, emerged in late 2019 from China, ${ }^{1}$ it spread fast all over the world. Italy was the first Western country that faced this treacherous infection, which had dramatic implications on overall social, political, and economic activities.

Lombardy was the Italian region hardest hit by the pandemic, and Bergamo, with its Papa Giovanni XXIII Hospital, was among the first and most affected centers worldwide. ${ }^{8,14}$ As first-line healthcare professionals, we neurosurgeons, along with other specialists, found ourselves at the forefront of this "infectious tsunami." Our neurosurgical unit was among the first involved in the healthcare system reorganization required to deal with the pandemic.

On the one hand, it was necessary to provide the resources to fight the infection, while on the other hand, access to neurosurgical care had to be guaranteed. The regional organization into hub-and-spoke centers, together with an effective process of prioritization and triaging, helped us to maintain a high standard of neurosurgical care, reducing the likelihood of clinical negligence claims with respective legal and financial implications. This meant carefully choosing all surgical cases that could be postponed without danger to life or neurological worsening, and at the same time also deciding how to address treatment for patients whose conditions would have seriously worsened due to a prolonged wait. This triaging resulted in the correct allocation of the reduced available resources (e.g., oncall neurosurgeons and nursing staff, operating room [OR] availability, and unavailable ICU beds) to manage these patients in our spoke center (e.g., those with chronic subdural hematomas or myeloradicular compression) who did not need a bed in the ICU, which would otherwise have risked clogging the hub center. In cases of subarachnoid hemorrhage with acute hydrocephalus, we first stabilized the patient (i.e., placed an external ventricular drainage system) and sent them to the reference hub center for major surgery or endovascular treatment.

The organizational and logistical choices that have been made allowed us to not ration patient treatment, thus avoiding collateral damage. The maintenance of a high standard of care also helped healthcare workers avoid the psychological distress that results from actions, or inactions, that violate an individual worker's ethical code.

We preserved a baseline activity of the outpatient clinic during the worst period and promptly reopened when the situation gradually improved. This was fundamental, because in the first weeks of the lockdown, we noticed an increase in advanced pathologies (e.g., lumbar disc herniation with cauda equina syndrome $\left.{ }^{14}\right)$, probably due to the fear of entering the hospital and incurring a viral infection. Many articles have addressed the effect on outpatient clinics, ${ }^{15}$ the virus effect on the central nervous system, ${ }^{16}$ the preparation of the OR for emergency surgeries for COVID-19 patients, ${ }_{17}^{17}$ and also studies on algorithms for scheduling neurosurgical cases during the pandemic..$^{10}$

Other authors have proposed a dynamic resource allocation to restrict OR access according to the current surge level of the virus. ${ }^{18}$ The proposed volume-limiting approach seems fluid and allows surgeons to triage on a case-by-case basis, allowing more flexible operative plans based on the surge level, OR availability, and need for emergency cases. However, the high rate and speed of transmission and virulence of COVID-19, coupled with the steeply increasing number of patients presenting to our emergency department (Fig. 1), did not allow us to apply a plan for progressive reduction of surgical and office activities. Moreover, since almost $75 \%$ of the neurosurgical staff was quickly redeployed to the newborn COVID-19 wards, the time for decisions and reorganization was limited.

According to others ${ }^{19-21}$ and to our own experience, focused and continued training of healthcare professionals through courses to treat patients of the pandemic helped to build well-integrated teams and provide appropriate care to COVID-19 patients. The courses should provide up-todate diagnostic-therapeutic guidelines for all staff and are fundamental in the management of a newly discovered disease, where treatment is still widely unknown. After many rotations, training courses, and daily clinical practice, all physicians efficiently improved their clinical skills and patient management. Neurosurgeons were particularly appreciated because of their skills in teamwork, flexibility, and natural attitude toward facing urgencies.

The COVID-19 pandemic proved able to overwhelm healthcare systems and concomitantly put in danger doctors and nurses. About $10 \%$ of the reported cases in China and Italy have been among healthcare workers. ${ }^{22}$ By April 2020, among the infected people in Italy more than 10,000 were healthcare workers, their number was stably increasing during those weeks, and more than 100 physicians died as a consequence of their assistance to COVID-19 patients. ${ }^{23}$

Among our staff, appropriate selection of neurosurgeons assigned to the COVID-19 wards effectively reduced the risk of contagion and its ensuing complications. Indeed, an investigation performed in our hospital at the beginning of May 2020 revealed that the asymptomatic positive rate among neurosurgeons was $26 \%$ (quite comparable to the general hospital rate). However, the rate of positive serological tests was very high compared with that in other Italian cities, reflecting the extremely elevated contagion rate that occurred in Bergamo. 


\section{Conclusions}

The COVID-19 experience saw us using all our strength to fight a worldwide crisis that has never been seen before. With this article, we want to pass on the following lessons we have learned so far:

- Rationalize resources through the creations of hub-andspoke centers, to concentrate the major forces working on the epidemic without forgetting to treat patients with urgent needs;

- Obtain PPE for all professional workers;

- Free up beds in the wards to become ICU beds and reallocate resources normally dedicated to other services (e.g., ventilators and monitors);

- Train staff to the new needs, providing up-to-date diagnostic and therapeutic guidelines;

- Select healthcare professionals who are at low risk of developing severe disease to assist COVID-19-positive patients;

- Cancel nonessential surgical cases to avoid putting at risk those patients who can have their treatment postponed without neurological risk;

- Swab all healthcare workers and patients before hospitalization. Patients requiring treatment need to be divided according to their test results (with clean and dirty tracks) to limit the spread of the disease at the hospital level. On the same basis, healthcare professionals should be tested;

- Manage patients at home. To limit patient access to healthcare facilities, when possible, patients should be managed by phone or by telemedicine;

- Share clinical data to benefit from the new therapeutic and diagnostic advantages.

\section{References}

1. Zhu N, Zhang D, Wang W, et al. A novel coronavirus from patients with pneumonia in China, 2019. N Engl J Med. 2020; 382(8):727-733.

2. Spina S, Marrazzo F, Migliari M, et al. The response of Milan's Emergency Medical System to the COVID-19 outbreak in Italy. Lancet. 2020;395(10227):e49-e50.

3. Grasselli G, Pesenti A, Cecconi M. Critical care utilization for the COVID-19 outbreak in Lombardy, Italy: early experience and forecast during an emergency response. JAMA. 2020;323(16):1545-1546.

4. Bernucci C, Brembilla C, Veiceschi P. Effects of the COVID-19 outbreak in Northern Italy: perspectives from the Bergamo Neurosurgery Department. World Neurosurg. 2020;137:465-468.e1.

5. Agosti E, Giorgianni A, Pradella R, Locatelli D. Coronavirus disease 2019 (COVID-19) outbreak: single-center experience in neurosurgical and neuroradiologic emergency network tailoring. World Neurosurg. 2020;138:548-550.

6. Cenzato M, DiMeco F, Fontanella M, et al. Editorial. Neurosurgery in the storm of COVID-19: suggestions from the Lombardy region, Italy (ex malo bonum). J Neurosurg. 2020; 133(1):33-34.

7. Perin A, Servadei F, DiMeco F. May we deliver neuro-oncology in difficult times (e.g. COVID-19)? J Neurooncol. 2020; 148(1):203-205.

8. Buoro S, Di Marco F, Rizzi M, et al. Papa Giovanni XXIII Bergamo Hospital at the time of the COVID-19 outbreak: letter from the warfront .... Int J Lab Hematol. 2020;42(suppl 1):8-10.

9. Fagiuoli S, Lorini FL, Remuzzi G. Adaptations and lessons in the province of Bergamo. N Engl J Med.2020;382(21):e71.
10. Burke JF, Chan AK, Mummaneni V, et al. Letter: The coronavirus disease 2019 global pandemic: a neurosurgical treatment algorithm. Neurosurgery. 2020;87(1):E50-E56.

11. Prall JA, Davis JD, Jenkins NR. Editorial. Community practice in the time of COVID-19. J Neurosurg. 2020;133(1):20-21.

12. Castelnuovo P, Turri-Zanoni M, Karligkiotis A, et al. Skullbase surgery during the COVID-19 pandemic: the Italian Skull Base Society recommendations. Int Forum Allergy Rhinol. 2020;10(8):963-967.

13. Tong X, Yang Y.Editorial. Lessons learned: special precautions for performing emergency cerebrovascular procedures amid the COVID-19 pandemic. J Neurosurg. 2020;133(1):22-23.

14. Brembilla C, Veiceschi P, Fanti A, et al. The eclipse of degenerative spine pathology during COVID-19 pandemic. Neurospine. 2020;17(2):354-356.

15. Chen Y, Pradhan S, Xue S. What are we doing in the dermatology outpatient department amidst the raging of the 2019 novel coronavirus? J Am Acad Dermatol. 2020;82(4):1034.

16. Baig AM, Khaleeq A, Ali U, Syeda H. Evidence of the COVID-19 virus targeting the CNS: tissue distribution, hostvirus interaction, and proposed neurotropic mechanisms. ACS Chem Neurosci. 2020;11(7):995-998.

17. Ti LK, Ang LS, Foong TW, Ng BSW. What we do when a COVID-19 patient needs an operation: operating room preparation and guidance. Can J Anaesth. 2020;67(6):756-758.

18. Bernstein M. Editorial. Neurosurgical priority setting during a pandemic: COVID-19. J Neurosurg. 2020;133(1):18-19.

19. Huh S. Editorial. How to train health personnel to protect themselves from SARS-CoV-2 (novel coronavirus) infection when caring for a patient or suspected case. J Educ Eval Health Prof. 2020;17:10.

20. Levi V, Risso A, Egidi M. Letter: The resiliency of the neurosurgeon in the midst of COVID-19 pandemic storm: the Italian experience from the frontline. Neurosurgery. 2020;87(2): E227-E228.

21. Mathiesen T, Arraez M, Asser T, et al. A snapshot of European neurosurgery December 2019 vs. March 2020: just before and during the Covid-19 pandemic. Acta Neurochir (Wien). 2020;162(9):2221-2233.

22. Tsermoulas G, Zisakis A, Flint G, et al. Challenges to neurosurgery during the coronavirus disease 2019 (COVID-19) pandemic. World Neurosurg. 2020;139:519-525.

23. Livingston E, Bucher K. Coronavirus disease 2019 (COVID-19) in Italy. JAMA. 2020;323(14):1335.

\section{Disclosures}

The authors report no conflict of interest concerning the materials or methods used in this study or the findings specified in this paper.

\section{Author Contributions}

Conception and design: Bernucci, Fanti. Acquisition of data: Bernucci, Fanti, Veiceschi, Brembilla. Analysis and interpretation of data: Bernucci, Fanti, Veiceschi. Drafting the article: Bernucci, Fanti, Veiceschi. Critically revising the article: Bernucci, Fanti. Reviewed submitted version of manuscript: Bernucci, Fanti. Approved the final version of the manuscript on behalf of all authors: Bernucci. Statistical analysis: Veiceschi. Administrative/ technical/material support: Fanti, Brembilla. Study supervision: Bernucci, Costi, Sicignano, Brembilla.

\section{Supplemental Information \\ Videos}

Video Abstract. https://vimeo.com/476318172.

\section{Correspondence}

Claudio Bernucci: ASST Papa Giovanni XXIII Hospital, Bergamo, Italy. cbernucci@asst-pg23.it. 